\title{
THE ROLE OF SUBSURFACE FLOWS IN SOLAR SURFACE CONVECTION: MODELING THE SPECTRUM OF SUPERGRANULAR AND LARGER SCALE FLOWS
}

\author{
J. W. LORD ${ }^{1}$, R. H. CAMERON ${ }^{2}$, M. P. RAST ${ }^{1}$, M. RemPel ${ }^{3}$, AND T. Roudier ${ }^{4}$ \\ ${ }^{1}$ Department of Astrophysical and Planetary Sciences, Laboratory for Atmospheric and Space Physics, \\ University of Colorado, Boulder, CO 80309, USA; mark.rast@lasp.colorado.edu \\ ${ }^{2}$ Max-Planck-Institut für Sonnensystemforschung, Justus-von-Liebig-Weg 3, D-37077 Göttingen, Germany \\ ${ }^{3}$ High Altitude Observatory, National Center for Atmospheric Research, Boulder, CO 80307, USA \\ ${ }^{4}$ Institut de Recherche en Astrophysique et Planétologie, Université de Toulouse, Centre national de la recherche scientifique (CNRS), F-31400 Toulouse, France \\ Received 2013 December 23; accepted 2014 July 7; published 2014 September 2
}

\begin{abstract}
We model the solar horizontal velocity power spectrum at scales larger than granulation using a two-component approximation to the mass continuity equation. The model takes four times the density scale height as the integral (driving) scale of the vertical motions at each depth. Scales larger than this decay with height from the deeper layers. Those smaller are assumed to follow a Kolmogorov turbulent cascade, with the total power in the vertical convective motions matching that required to transport the solar luminosity in a mixing length formulation. These model components are validated using large-scale radiative hydrodynamic simulations. We reach two primary conclusions. (1) The model predicts significantly more power at low wavenumbers than is observed in the solar photospheric horizontal velocity spectrum. (2) Ionization plays a minor role in shaping the observed solar velocity spectrum by reducing convective amplitudes in the regions of partial helium ionization. The excess low wavenumber power is also seen in the fully nonlinear three-dimensional radiative hydrodynamic simulations employing a realistic equation of state. This adds to other recent evidence suggesting that the amplitudes of large-scale convective motions in the Sun are significantly lower than expected. Employing the same feature tracking algorithm used with observational data on the simulation output, we show that the observed low wavenumber power can be reproduced in hydrodynamic models if the amplitudes of large-scale modes in the deep layers are artificially reduced. Since the large-scale modes have reduced amplitudes, modes on the scale of supergranulation and smaller remain important to convective heat flux even in the deep layers, suggesting that small-scale convective correlations are maintained through the bulk of the solar convection zone.
\end{abstract}

Key words: convection - Sun: granulation - Sun: interior - Sun: photosphere - turbulence

Online-only material: color figures

\section{INTRODUCTION}

Solar supergranulation is observed as horizontal divergent flow within magnetic network boundaries (Leighton et al. 1962; Simon \& Leighton 1964), either by Doppler imaging away from the disk center (Hathaway et al. 2000) or by correlation (November et al. 1988; DeRosa \& Toomre 2004; Meunier et al. 2007) or structure tracking near the disk center (Roudier et al. 1999, 2012). The power spectrum of the horizontal motions shows a characteristic peak at horizontal scales ranging from approximately $20 \mathrm{Mm}$ to $50 \mathrm{Mm}$, and the motions at these scales are identified as supergranulation. There is a dramatic drop in spectral power for scales larger than supergranulation with very weak giant cell flows only recently confirmed by observations (Hathaway et al. 2013).

The physical origin of the supergranular length scale remains a mystery. Suggestions range from possible dynamical effects of the second ionization of helium (Leighton et al. 1962; Simon \& Leighton 1964; November et al. 1981) to spatial correlation or self-organization of granular flows (Rieutord et al. 2000; Rast 2003; Crouch et al. 2007). Radiative hydrodynamic simulations of solar surface convection fail to yield clear evidence for supergranulation, even in very large domains spanning up to $96 \mathrm{Mm} \times 96 \mathrm{Mm}$ in width and $20 \mathrm{Mm}$ in depth (Stein et al. 2009; Ustyugov 2010). Recent simulations in even larger domains of up to $196 \times 196 \times 49 \mathrm{Mm}^{3}$ suggest that the domain depth and the consequent stratification captured by the simulation may be as critical as the domain width (J. W. Lord 2014, in preparation). Based on these broad and deep simulations of solar surface convection we have developed a model of the convective velocity spectrum that reproduces the simulation spectrum and provides insight into how the deep convective flows help build the observed photospheric spectrum.

The model assumes that, at each depth, vertical motions are driven at scales four times the local density scale height. The amplitudes of smaller scale motions are taken to be consistent with the spectrum of unstratified and incompressible turbulence. Larger scale vertical motions imprint from below with reduced amplitude and are observed as primarily horizontal flows at the surface (Spruit et al. 1990). In other words, modes with wavelengths smaller than the integral (driving) scale are assumed to have amplitudes that follow the spectrum of isotropic homogeneous turbulence given by Kolmogorov (1941), while vertical motions of scales larger than the integral scale are assumed to decay with height from their driving depth. The integrated power of the vertical velocity is determined using a mixing length model of energy transport, and the spectrum of horizontal velocity follows from the vertical velocity spectrum at each depth by mass continuity.

Key scalings in the model are verified using the radiative hydrodynamic simulations of J. W. Lord 2014 (in preparation), and the simplified model spectra agree with those of the simulations over a wide range of wavenumbers. They also match observations at supergranular scales. However, power at lower 
wavenumbers, in both the model and radiative hydrodynamic simulation spectra, significantly exceeds that observed. This suggests either that large-scale flows deep in the solar convection zone are weaker than predicted by convection simulations or that rotation and the consequent near surface shear layer, not captured in our studies, plays a decisive role in masking large-scale motions. We note, however, that recent helioseismic observations (Hanasoge et al. 2010, 2012) and global scale numerical simulations, with and without a near surface shear layer (H. Hotta et al. 2014, in preparation), also suggest that largescale convection in the Sun is weaker than numerical models predict. It is possible that magnetic fields play a role given that convection in the Sun is fundamentally magnetized. Preliminary studies of radiative magnetohydrodynamic simulations (J. W. Lord 2014, in preparation) in very large domains show some suppression of low wavenumber power in highly magnetized solutions, though the mechanism is still under investigation and the effect so far appears insufficient to explain solar observations. In this paper, we focus on strictly hydrodynamic effects to elucidate the important role of stratification and the secondary influence of ionization in shaping the photospheric horizontal velocity power spectrum at supergranular and larger scales.

In Section 2 we describe the simplified two-component continuity balance on which our model is based and transform the balance equations into relationships between the vertical and horizontal velocity spectra. We use these relationships to identify the driving scale of the modes and demonstrate that these relationships hold in fully compressible hydrodynamic simulations. In Section 3 we describe the construction of the mixing length atmosphere which sets the amplitude of the model spectrum, identify two possible decay rates for the largescale modes, and explicitly outline the model steps employed in the construction of the surface horizontal velocity spectrum. In Section 4 we test the components of the model spectrum against the full radiative hydrodynamic solutions and verify that the model can reproduce the shape of the spectrum produced by those simulations. In Section 5 we discuss the results of the model spectrum, focusing on the spectrum at supergranular scales and larger. We show, using feature tracking, that for scales larger than supergranulation, the radiative hydrodynamic spectra can only match the observations when the convective forcing is removed entirely below $10 \mathrm{Mm}$. We conclude in Section 6 with a discussion of the broader implications of the observed weak low wavenumber amplitudes to our understanding of deep solar convection.

\section{MASS CONTINUITY AND THE EFFECTS OF STRATIFICATION}

We use the equation of mass continuity to examine how stratification affects flow velocity. Explicitly,

$$
\begin{aligned}
\frac{\partial \rho}{\partial t}+\nabla \cdot(\rho \mathbf{u})= & \frac{\partial \rho}{\partial t}+\rho\left(\frac{\partial u_{x}}{\partial x}+\frac{\partial u_{y}}{\partial y}+\frac{\partial u_{z}}{\partial z}\right)+u_{x} \frac{\partial \rho}{\partial x} \\
& +u_{y} \frac{\partial \rho}{\partial y}+u_{z} \frac{\partial \rho}{\partial z}=0
\end{aligned}
$$

where $\rho$ is the mass density, $\mathbf{u}$ is the fluid velocity, and subscripts $x, y$, and $z$ indicate components in Cartesian coordinates. We ignore curvature, and take gravity, and thus increasing density in the stratified domain, to be in the positive $z$ direction.

In the solar convection zone we can make a number of further simplifying assumptions. Since we are looking for the statistically steady velocity amplitudes over time periods much longer than the convective turnover time, we take $(\partial \rho / \partial t) \rightarrow 0$. Moreover, we know from hydrodynamic simulations that the horizontal gradients of the density are small compared to the vertical stratification below the first few hundred kilometers beneath the solar photosphere, so $u_{x}(\partial \rho / \partial x)$ and $u_{y}(\partial \rho / \partial y)$ are ignored. Together these assumptions yield an anelastic-like continuity equation (Gough 1969) that maintains the steady state stratification by balancing the vertical advection of mass with the divergence of the flow,

$$
\nabla_{h} \cdot \mathbf{u}_{h}=-\frac{\partial u_{z}}{\partial z}-\frac{u_{z}}{H_{\rho}},
$$

where $\mathbf{u}_{h}=u_{x} \hat{i}+u_{y} \hat{j}, \nabla_{h}=\hat{i}(\partial / \partial x)+\hat{j}(\partial / \partial y)$, and $H_{\rho}=$ $((1 / \rho)(d \rho / d z))^{-1}$ is the density scale height.

This form of the continuity equation suggests two possible flow regimes: for $\left(\partial u_{z} / \partial z\right) \gg\left(u_{z} / H_{\rho}\right)$ the motions may be considered nearly divergenceless and isotropic whereas for $\left(\partial u_{z} / \partial z\right) \ll\left(u_{z} / H_{\rho}\right)$ the stratification is most important in determining the flow component speeds. Heuristically, smallscale overturning eddies would fall in the first regime, while eddies larger than the local scale height would fall in the second, with the largest isotropic eddies increasing in size with depth as the density scale height increases. Thus we expect the dominant balance in Equation (2) to depend on the length scale of the flow and depth within the convection zone.

Maintaining the mean stratification in a statistically steady stratified convective flow requires that most of the mass must overturn as the fluid rises through one scale height; over each scale height, the density of rising fluid must decrease by a factor of $1 / e$, implying that $1-1 / e$ of the mass must overturn. Similarly, downwelling fluid must entrain mass at this rate. If the flow geometry is approximated by vertical cylinders of radius $r$ and height $H_{\rho}$, then for all of the mass to overturn within one scale height, $2 \pi r H_{\rho} \rho u_{h}=\pi r^{2} \rho u_{z}$. This yields a characteristic horizontal scale for the motions (Nordlund et al. 2009)

$$
r=2 \alpha H_{\rho} \frac{u_{h}}{\left|u_{z}\right|},
$$

where $\alpha$ is a factor of order 1 and includes a weak dependence on geometry and the $1 / e$ fraction of the mass that does not overturn. We take this length scale to be the crossover between those motions that feel the stratification and those that do not. We demonstrate in the next section that such a crossover is seen in the spectra of three-dimensional simulations. This length scale is also the integral scale of the velocity spectrum in solar-like hydrodynamic simulations (Stein et al. 2009), and henceforth we refer to it as the driving or integral scale of the convection.

\subsection{The Spectra of Horizontal and Vertical Motions}

Equation (2) can be written as

$$
i \mathbf{k}_{h} \cdot \tilde{\mathbf{u}}_{h}=-\frac{\partial \tilde{u}_{z}}{\partial z}-\frac{\tilde{u}_{z}}{H_{\rho}},
$$

where the overlying tildes indicate the complex Fourier amplitudes resulting from a two-dimensional horizontal Fourier transform at each depth $z$ and $\mathbf{k}_{h}$ is the horizontal mode wave vector. By squaring both sides and taking the two limits of Equation (4), we can define a relationship between the power in horizontal and vertical motions without directly solving for 


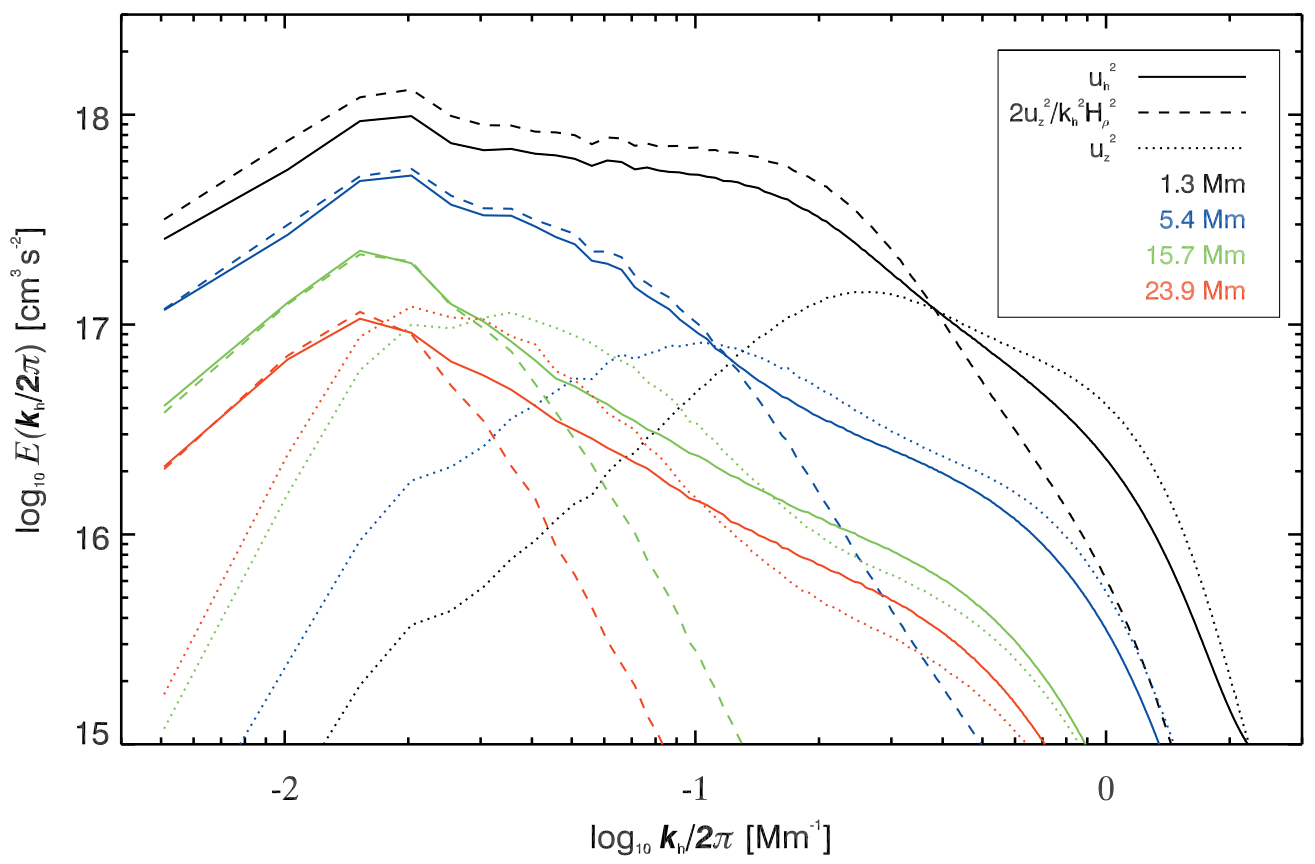

Figure 1. Horizontal velocity power spectra (solid curves) from hydrodynamic simulations using a Saha equation of state (see Sections 3.1 and 5.2 for details) at four depths: black 1.3 Mm, blue 5.4 Mm, green $15.7 \mathrm{Mm}$, and red 23.9 Mm below the photosphere. The dashed and dotted curves show the horizontal velocity spectra deduced from the vertical velocity based on Equations (5) and (6), respectively. The velocity is averaged over 30 minutes before computing these spectra to remove $p$-modes. This averaging also reduces power in high wavenumber convective motions.

(A color version of this figure is available in the online journal.)

the phases of the modes. For modes smaller than the integral scale, we take the limit $\left(\partial \tilde{u}_{z} / \partial z\right) \gg\left(\tilde{u}_{z} / H_{\rho}\right)$, while for larger scale modes we take $\left(\partial \tilde{u}_{z} / \partial z\right) \ll\left(\tilde{u}_{z} / H_{\rho}\right)$. Even in these limits, defining the relationship between vertical and horizontal power is difficult for two reasons: when squaring Equation (4), the cross terms between horizontal modes $\tilde{u}_{x}$ and $\tilde{u}_{y}$ on the left side do not have an a priori known form, and the vertical derivative on the right-hand side cannot be simply related to the wavenumber of the horizontal Fourier modes.

To proceed we make simplifying assumptions that we have empirically verified to hold in stratified (J. W. Lord 2014, in preparation) and incompressible turbulence simulations (Mininni et al. 2006) as appropriate. At small scales, the flow is nearly isotropic and homogeneous, with unstratified homogeneous and isotropic turbulence simulations showing that $k_{x}^{2} \tilde{u}_{x} \tilde{u}_{x}^{*} \approx k_{y}^{2} \tilde{u}_{y} \tilde{u}_{y}^{*} \approx\left(\partial \tilde{u}_{z} / \partial z\right)\left(\partial \tilde{u}_{z}^{*} / \partial z\right)$, which together with incompressibility, yields $\left(\partial \tilde{u}_{z} / \partial z\right)\left(\partial \tilde{u}_{z}^{*} / \partial z\right) \approx(1 / 4) k_{h}^{2} \tilde{\mathbf{u}}_{h} \cdot \tilde{\mathbf{u}}_{h}^{*}$. The simulations also suggest a relationship between the vertical and horizontal gradients, $\left(\partial \tilde{u}_{z} / \partial z\right)\left(\partial \tilde{u}_{z}^{*} / \partial z\right) \approx(1 / 4) k_{h}^{2} \tilde{u}_{z} \tilde{u}_{z}^{*}$, and together these yield a relationship between horizontal and vertical power:

$$
\tilde{\mathbf{u}}_{h} \cdot \tilde{\mathbf{u}}_{h}^{*}=\tilde{u}_{z} \tilde{u}_{z}^{*},
$$

where $\tilde{\mathbf{u}}_{h} \cdot \tilde{\mathbf{u}}_{h}^{*}=\tilde{u}_{x} \tilde{u}_{x}^{*}+\tilde{u}_{y} \tilde{u}_{y}^{*}$. At large scales, the cross terms, which result from squaring the left-hand side of Equation (4), are measured in stratified simulations to be small and are set to zero. This implies that $k_{x}^{2} \tilde{u}_{x} \tilde{u}_{x}^{*}+k_{y}^{2} \tilde{u}_{y} \tilde{u}_{y}^{*} \approx \tilde{u}_{z} \tilde{u}_{z}^{*} / H_{\rho}^{2}$, and the horizontal and vertical power in the modes are related as

$$
\tilde{\mathbf{u}}_{h} \cdot \tilde{\mathbf{u}}_{h}^{*}=\frac{2}{k_{h}^{2} H_{\rho}^{2}} \tilde{u}_{z} \tilde{u}_{z}^{*}
$$

where $k_{h}^{2}=k_{x}^{2}+k_{y}^{2}$.
Finally, without approximation, the driving scale that separates these two behaviors (Equation (3)) can be rewritten as

$$
\lambda_{h}=4 \alpha H_{\rho} \frac{u_{h}}{\left|u_{z}\right|},
$$

where $\lambda_{h}=2 \pi / k_{h}$ is the wavelength of the Fourier mode corresponding to a convective cell diameter of $d=2 r$. By taking $\alpha$ and $u_{h} / u_{z} \approx 1$ we approximate the driving scale as $\lambda_{h} \approx 4 H_{\rho}$. It is on the basis of these relationships (Equation (5) at small scales and Equation (6) at large scales with the crossover between them given by the driving scale $\lambda_{h}=4 H_{\rho}$ at each depth) that we calculate the horizontal velocity power spectrum from the vertical.

Analysis of large-scale radiative hydrodynamic simulations of solar convection (for details, see Section 5.1 and J. W. Lord 2014 , in preparation) helps validate these relationships. Below 1.3 Mm beneath the photosphere, the mass continuity in the simulations matches the anelastic balance (Equations (2) and (4)) to within a few percent. Near the photosphere, this balance breaks down because of fluid compressibility, particularly at high wavenumbers. We thus restrict our model analysis to depths below $1.3 \mathrm{Mm}$. At low wavenumbers, $p$-mode contributions can still be important at the shallowest depths. We remove these when comparing the numerical simulations to the model by averaging the simulation velocities over 30 minutes. This averaging also reduces the amplitude of the high wavenumber convective motions, but preserves the relationships between horizontal and vertical flows of Equations (5) and (6). This is illustrated by Figure 1, in which the horizontal velocity spectra measured at several depths in a solar-like radiative hydrodynamic simulation are plotted. Overplotted are the horizontal velocity spectra deduced from the vertical velocity spectra of the simulation at the same depths using Equations (5) and (6) (dotted and dashed line 


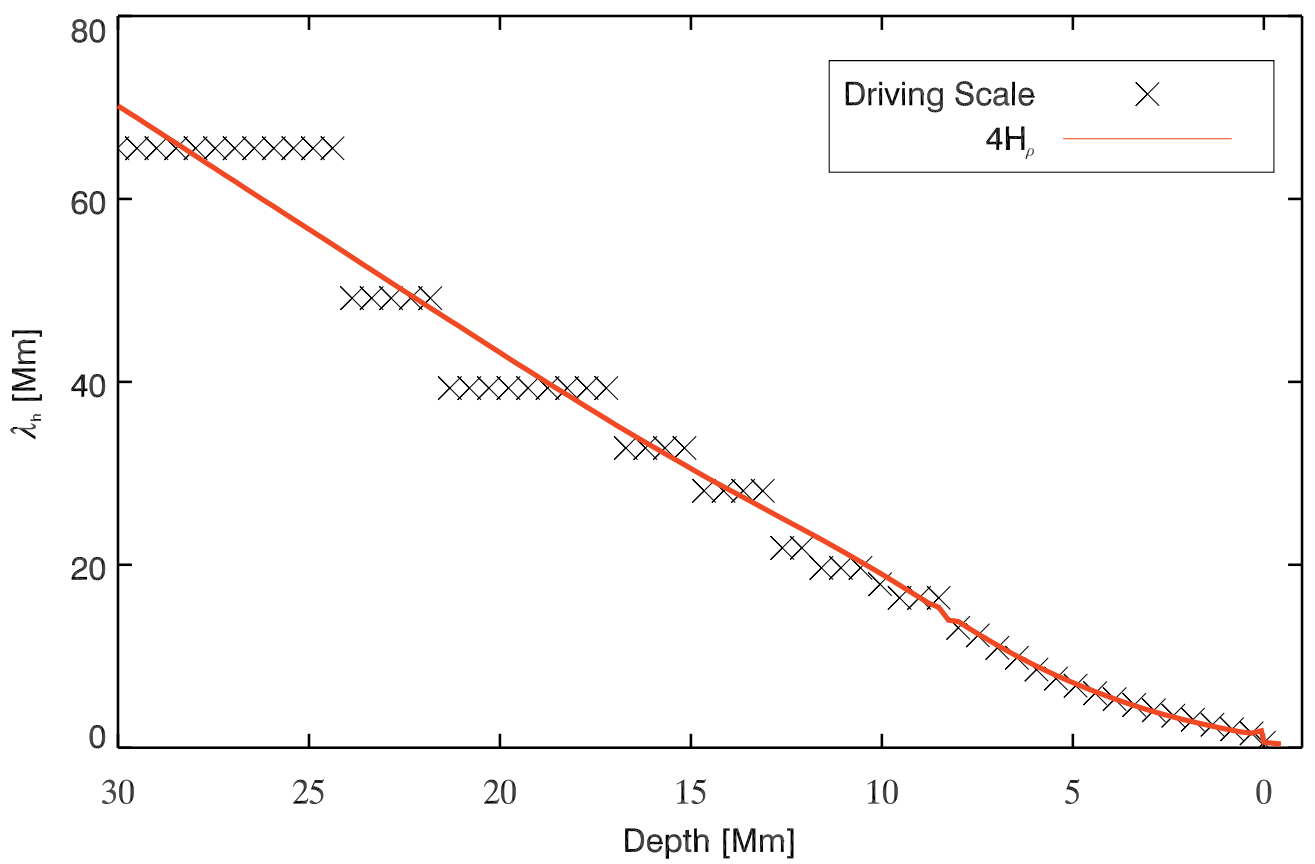

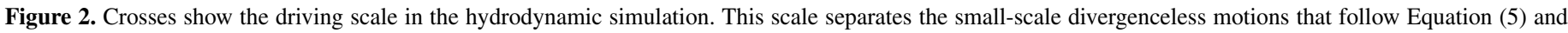

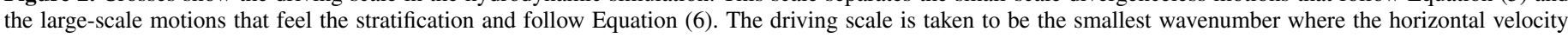
spectrum begins to systematically diverge from Equation (6). The solid (red) curve shows $4 H_{\rho}$ from the hydrodynamic simulation.

(A color version of this figure is available in the online journal.)

styles, respectively). The two-component reconstruction of the horizontal velocity spectrum from the vertical reproduces the shape and amplitude of the actual spectrum quite well. Moreover, the driving scale estimate of $4 H_{\rho}$ is in good agreement with the crossover between the two behaviors. Plotted in Figure 2 is the crossover wavenumber as a function of depth (defined as the smallest wavenumber in the simulations for which the balance in Equation (6) begins to fail, meaning that the difference between the two terms at neighboring larger horizontal wavenumbers is increasingly large). For comparison, $4 H_{\rho}$ is overplotted in red. They are in good agreement. Note that the discontinuities in the measured values are due to the finite spectral resolution of the simulation; many depths in the simulation appear to have the same crossover scale because there are no modes that can discriminate between them.

\section{MODEL COMPONENTS}

Having verified the two-component continuity balance, we construct the horizontal spectrum of horizontal motions from the horizontal spectrum of the vertical velocity using the relationships derived. To do this, we must model the vertical velocity spectrum at each depth. This depends on the driving (integral) scale at that depth, the spectrum of the small-scale motions, and the decay rate of the large-scale modes that are driven below the height of consideration. We have already defined the driving scale as $4 H_{\rho}$, and we choose the spectrum of the higher wavenumber motions to follow a turbulent cascade with a $k^{-5 / 3}$ Kolmogorov slope. The Kolmogorov spectrum does not match the spectrum of motions in the hydrodynamic simulations exactly, but we use it in the model for simplicity and in place of an ad hoc fit to the simulations, which themselves may not match the spectrum of solar motions (see Section 4). The integrated spectral power is determined using the rms velocity of a mixing length model of solar convection (Section 3.1). Thus, the amplitudes of modes with scales larger than the driving scale are determined by their decay with height from the depth at which they were last driven (Section 3.2), and the remaining power (the rms velocity squared minus the power in large-scale modes) is distributed among all modes at the driving scale or smaller according to a Kolmogorov distribution.

\subsection{Mixing Length Transport by Small-scale Modes}

We employ a simplified hydrostatic mixing length atmosphere of pure hydrogen and helium in Saha equilibrium, integrating the mixing length equations (Prandtl 1925; Böhm-Vitense 1958) using the observed density and temperature of the solar photosphere as boundary conditions. We take the convective flux to be equal to the photospheric radiative output $(6.3 \times$ $10^{10} \mathrm{erg} \mathrm{cm}^{-2} \mathrm{~s}^{-1}$ ) as appropriate for efficient convection and note that this introduces an error in the lower portions of the model where, in the Sun, radiation transports a significant fraction of the energy flux. This error makes a small contribution to the excess model power at the largest scales (Section 4).

Explicitly, we solve the equation for the convective energy flux $F_{c}=(1 / 2) \rho v C_{p} T\left(l / H_{p}\right)\left(\nabla-\nabla^{\prime}\right)$ along with that of hydrostatic balance $(d P / d z)=-\rho g$. Here $\rho$ is the fluid density, $v$ is the velocity, $C_{p}$ is the specific heat at constant pressure, $T$ is the temperature, $\nabla$ is the mean temperature gradient, $\nabla^{\prime}$ is the temperature gradient within the convective cell, $P$ is the pressure, and $g=G m(z) / r(z)^{2}$ is the gravitational acceleration with $r(z)$ the distance from the Sun's center, $m(z)$ the mass within that radius, and $G$ the gravitational constant. We employ the equation of state $P=\rho k T / \mu$, where $k$ is the Boltzmann constant and $\mu$ is the mean molecular weight of the plasma, and assume that the convective motions are adiabatic, so that $\nabla^{\prime}=\nabla_{\mathrm{ad}}=\left.(\partial \ln T / \partial \ln P)\right|_{\mathrm{ad}}$, the adiabatic temperature gradient. Finally, the rms convective velocity is given by $v^{2}=(1 / 8) g Q\left(l^{2} / H_{p}\right)\left(\nabla-\nabla^{\prime}\right)$, where $l$ is the mixing length measured in units of the pressure scale height $H_{P}$. Note that $Q=1-\left.(\partial \ln \mu / \partial \ln T)\right|_{P}, C_{p}, \nabla_{\mathrm{ad}}$, and $\mu$ account for 


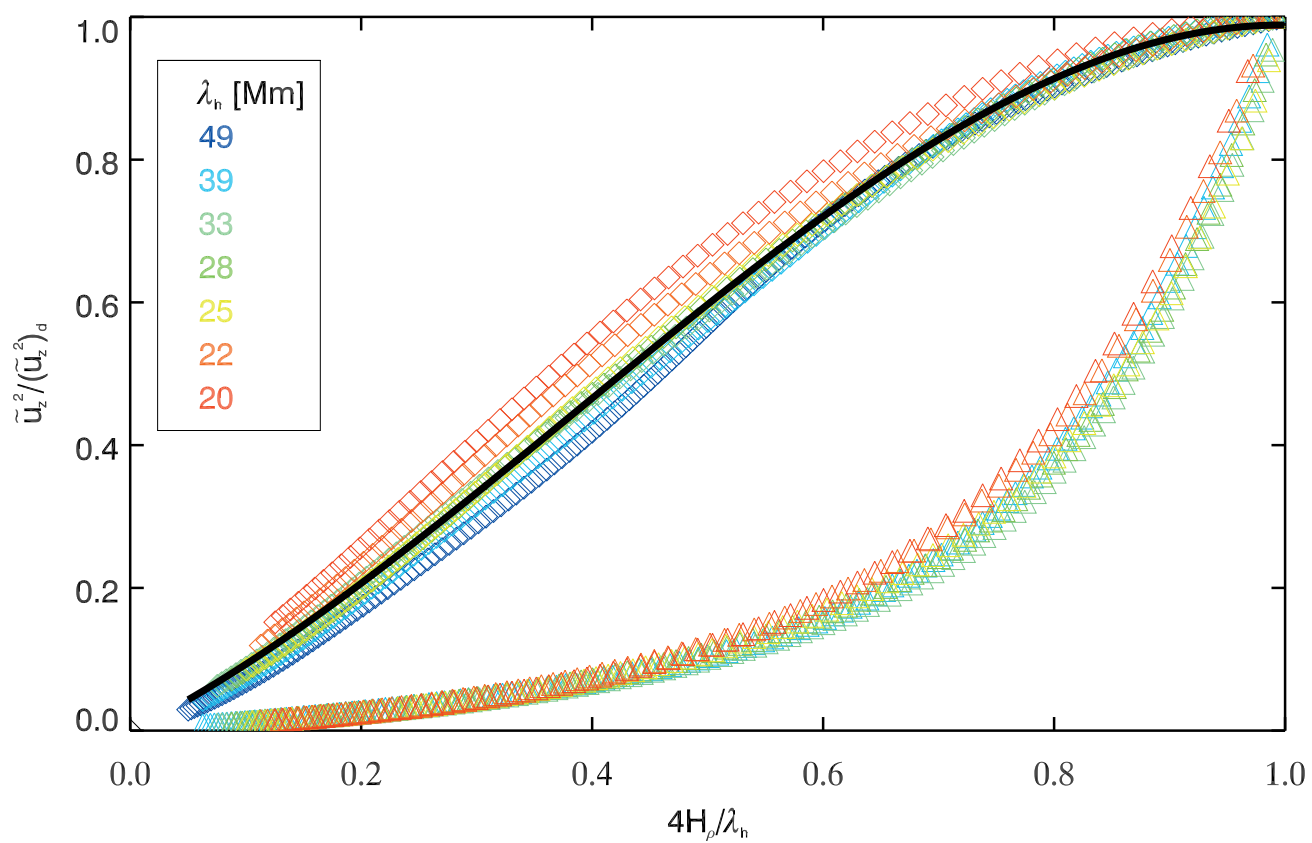

Figure 3. Diamonds show vertical velocity power from the driving depth, $\lambda_{h}=4 H_{\rho}$, up to $1.3 \mathrm{Mm}$ below the photosphere in the hydrodynamic simulation. The different colors show different modes from $20 \mathrm{Mm}$ (red) to $50 \mathrm{Mm}$ (purple). The black curve is the cubic polynomial fit to the observed decay rate. The triangles show the decay rate for a potential flow (Equation (9)) for the same range of modes.

(A color version of this figure is available in the online journal.)

the non-ideal effects of hydrogen and helium ionization (where the number density of each ionization state is determined in collisional equilibrium as a Saha balance).

The equations are integrated numerically from the photosphere downward, yielding the convective rms velocity and the local density scale height at each depth.

\subsection{Decay of Large-scale Modes with Height}

In all simulations of solar convection, the amplitude of the vertical velocity at low wavenumbers decreases toward the surface where granular scale convection is dominant. The rate of this decrease for the largest scale convective modes is a fundamental uncertainty in our understanding of solar convection. While global simulations predict giant cell convection throughout much of the convection zone (e.g., Miesch et al. 2008), surface observations have only very recently found evidence for weak flows at these scales (Hathaway et al. 2013). Numerical simulations have difficulty directly addressing the photospheric amplitude of large-scale motions. They are either of limited extent in depth (Stein et al. 2009; Ustyugov 2010; J. W. Lord 2014, in preparation) or do not capture the non-ideal and highly compressible nature of the uppermost layers (Miesch et al. 2008).

Because of these uncertainties, we examine two possible vertical velocity amplitude decay profiles. Starting from the depth at which the wavelength of the mode exceeds the integral scale, we decay the modes either by approximating the flow as potential (van Ballegooijen 1986) or by using a cubic polynomial fit to the observed decay of modes with wavelengths between $20 \mathrm{Mm}$ and $50 \mathrm{Mm}$ in the hydrodynamic simulations (shown as diamonds in Figure 3). The potential flow approximation takes the flow to be irrotational, allowing a direct solution to the large-scale continuity balance, written as

$$
\frac{\partial \tilde{\phi}}{\partial z}=-k_{h} H_{\rho} \tilde{\phi}
$$

where $\phi$ is the velocity potential with $\tilde{u}_{z}=\partial \tilde{\phi} / \partial z$. This yields a profile for the velocity amplitude with height

$$
\tilde{u}_{z}(z)=\tilde{u}_{z}\left(z_{d}\right) \frac{H_{\rho}(z)}{H_{\rho}\left(z_{d}\right)} \exp \left[k_{h}^{2} \int_{z_{d}}^{z} H_{\rho}\left(z^{\prime}\right) d z^{\prime}\right],
$$

where $z_{d}$ is the driving depth. This velocity profile can be integrated numerically for any wavenumber $k_{h}$, the results of which are shown with triangles in Figure 3. The polynomial fit, on the other hand, approximates the decay of the modes by a single function determined from the hydrodynamic simulations (solid line in Figure 3). The fit groups the behavior of all modes between 20 and $50 \mathrm{Mm}$ together and is thus inadequate to reproduce the hydrodynamic simulation in detail (see Section 4). It is employed in the model because of its simplicity. The two schemes are quite different in form and together provide a test of the sensitivity of the model to this key unknown function.

\subsection{Construction of the Model Spectrum}

In summary, we construct the model surface horizontal velocity spectrum as follows. To calculate the spectrum of the vertical velocity we follow these steps.

1. Construct a mixing length model of the solar convection zone integrating from the photosphere downward to $200 \mathrm{Mm}$, the approximate depth of the solar convection zone.

2. Determine the wavelength of the largest scale mode allowed at the bottom of the model atmosphere, $\lambda_{h}=4 H_{\rho}$, and use this as the integral (driving) scale (i.e., the lowest wavenumber mode) in a $k^{-5 / 3}$ turbulent cascade. The highest wavenumber in the spectrum is taken to be the Nyquist frequency of the hydrodynamic simulations on which the model is based $(2 \pi / 384 \mathrm{~km}$, see Section 5.1), and the spectrum is normalized so that the integrated power is equal to the mixing length velocity squared at the bottom of the model atmosphere. 
3. Move one step up in the atmosphere (a grid spacing of $64 \mathrm{~km}$ is used to again match the hydrodynamic simulations). Decay modes with wavelengths longer than the local integral scale $\left(4 H_{\rho}\right)$ using one of the two decay functions discussed in Section 3.2. Compute the integrated power in the decaying modes and normalize the remaining $k^{-5 / 3}$ spectrum by the squared mixing length velocity minus the power in the decaying modes.

4. Repeat step 3 until the top of the model atmosphere is reached.

From the vertical velocity spectrum, the horizontal velocity spectrum at any height is computed using Equations (5) and (6).

\section{TESTING THE MODEL}

We used the model outlined above to compute the horizontal velocity spectrum at a depth of $1.3 \mathrm{Mm}$ below the solar photosphere (as previously discussed, model assumptions break down above this height and results from hydrodynamic simulations validate this spectra as a close approximation to the surface spectrum for supergranular and larger scale motions). The resulting spectrum is shown as a solid (red) curve in Figure 4(a). For clarity we show the spectrum obtained when employing the large-scale mode decay rate as measured in the hydrodynamic simulation only (we discuss the potential decay below). The spectrum has two notable low wavenumber features: monotonically increasing power at scales larger than supergranulation and a small plateau of power at supergranular scales. The monotonic increase of power to lower wavenumbers is not observed on the Sun. The horizontal velocity spectrum of solar motions shows decreasing power at scales larger than supergranulation (see Section 5.1). The small supergranular plateau extends from $\lambda_{h} \sim 20-30 \mathrm{Mm}$ (corresponding to $k / 2 \pi \sim 0.03-0.05 \mathrm{Mm}^{-1}$ in Figure 4(a)), matching supergranular scales in solar observations. The high wavenumber features of the spectrum, in particular the discontinuity at the $1.3 \mathrm{Mm}$ depth integral scale, occur at scales for which the model is ill-suited.

To test the sensitivity of the model to the mixing length atmosphere employed we compared the spectrum shown in Figure 4(a) to one computed using a more sophisticated nonlocal mixing length formulation (Christensen-Dalsgaard et al. 1996). The non-local formulation employs the OPAL (Rogers \& Iglesias 1992) equation of state and opacities, more carefully accounting for the chemical composition of the convection zone. Importantly, it also accounts for the transport of energy by radiation in the lower portion of the convection zone, which reduces the convective flux and consequent driving amplitudes there. The resulting horizontal velocity spectrum has a shape nearly identical to that computed using our simplified Saha balance. Only the largest scale mode shows any notable difference, with the amplitude of that mode somewhat reduced as it is the only mode driven in the lower third of the convection zone where the Christensen-Dalsgaard et al. (1996) convective velocities are weaker as a result of the more careful accounting of the radiative energy flux.

As there is no a priori expectation for the decay rate of the large-scale modes, this aspect of the model is more difficult to assess. We chose to compare the spectrum shown in Figure 4(a) (obtained using the large-scale mode decay rate measured in the hydrodynamic simulations) to one employing an analytic potential flow assumption (van Ballegooijen 1986) because the latter yields an exponential decay of the mode amplitudes (Equation (9)) and may thus represent a somewhat limiting
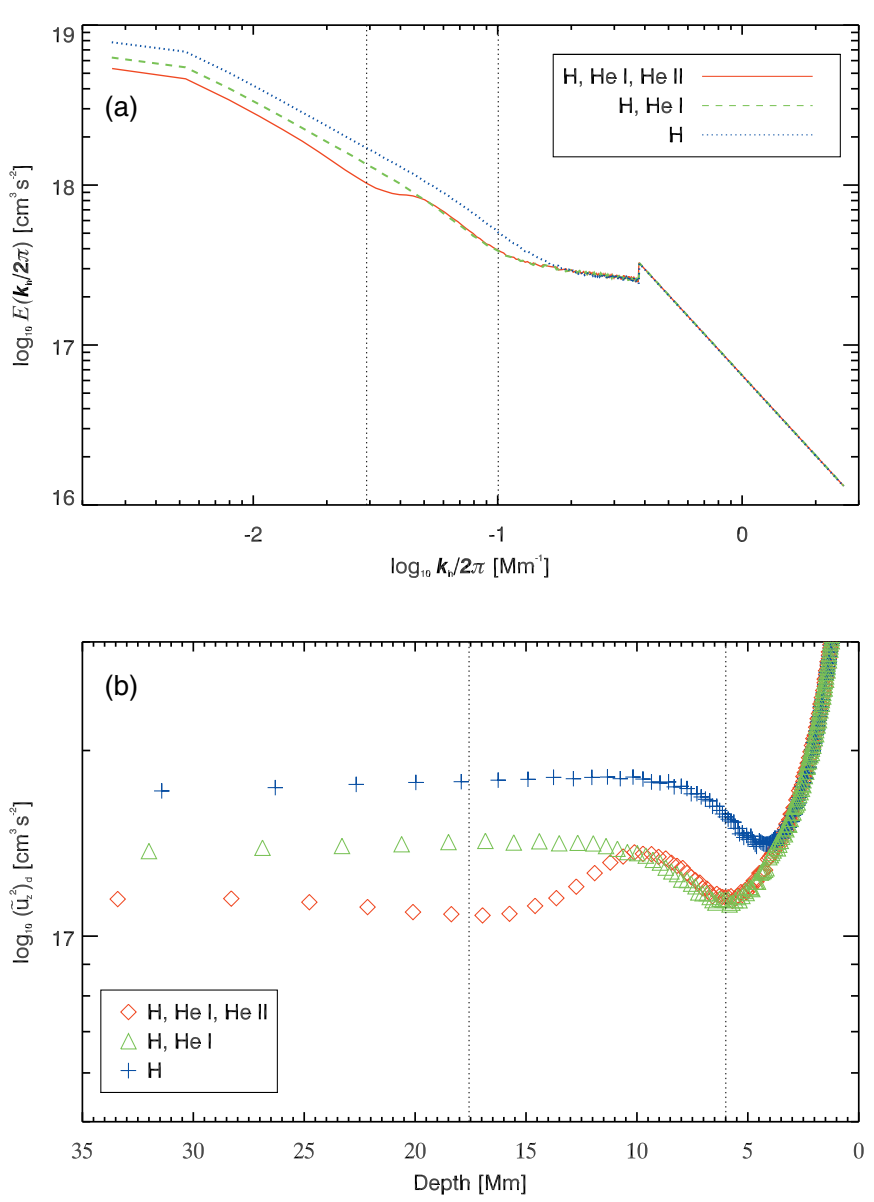

Figure 4. Spectra computed from the two-component model using the decay rate fit to the hydrodynamic simulations. We show the horizontal velocity spectrum at a depth of $1.3 \mathrm{Mm}$ in part (a) and the vertical velocity power at the driving depth in part (b). The solid (red) curve in part (a) and diamonds in part (b) (red) show the spectrum computed from a mixing length atmosphere with a Saha equation of state that includes $\mathrm{H}, \mathrm{He}$, and He II ionization; the dashed in (a) and triangles in (b) (green) are computed from an atmosphere with no He II ionization; and the dotted line in (a) and crosses in (b) (blue) are computed from an atmosphere with no He I or II ionization. The vertical dotted lines show the depths of $50 \% \mathrm{He}$ I $\left(6 \mathrm{Mm}\right.$ and $\left.k / 2 \pi=0.1 \mathrm{Mm}^{-1}\right)$ and He II $(17.5 \mathrm{Mm}$ and $k / 2 \pi=0.03 \mathrm{Mm}^{-1}$ ) ionization in part (b) and driving scale, where $\lambda_{h}=4 H_{\rho}$, at those depths in part (a).

(A color version of this figure is available in the online journal.)

case. The surface horizontal velocity spectrum computed with the exponential decay shows significant reduction in overall power, particularly at small scales, but quite similar shape at supergranular scales. At scales larger than supergranulation it exhibits a nearly identical monotonic increase of power to that seen in Figure 4(a). The relative amplitudes of low wavenumber modes are quite insensitive to the imposed mode decay function. The low wavenumber power distribution is primarily determined by the mode amplitudes at depth, with those amplitudes constrained by convective flux requirements of the model atmosphere.

Finally, we looked to validate the model using the threedimensional hydrodynamic simulations directly. When taking the driving scale $\left(4 H_{\rho}\right)$ and rms velocity amplitude directly from the simulation itself, rather than from a mixing length atmosphere, the model matches the horizontal velocity spectrum of simulation to within $10 \%$ over the wavenumber band $k / 2 \pi=0.02-0.15 \mathrm{Mm}^{-1}\left(\lambda_{h} \sim 7-50 \mathrm{Mm}\right)$ at all depths below 1.3 Mm. This is achieved, however, only by fitting the 


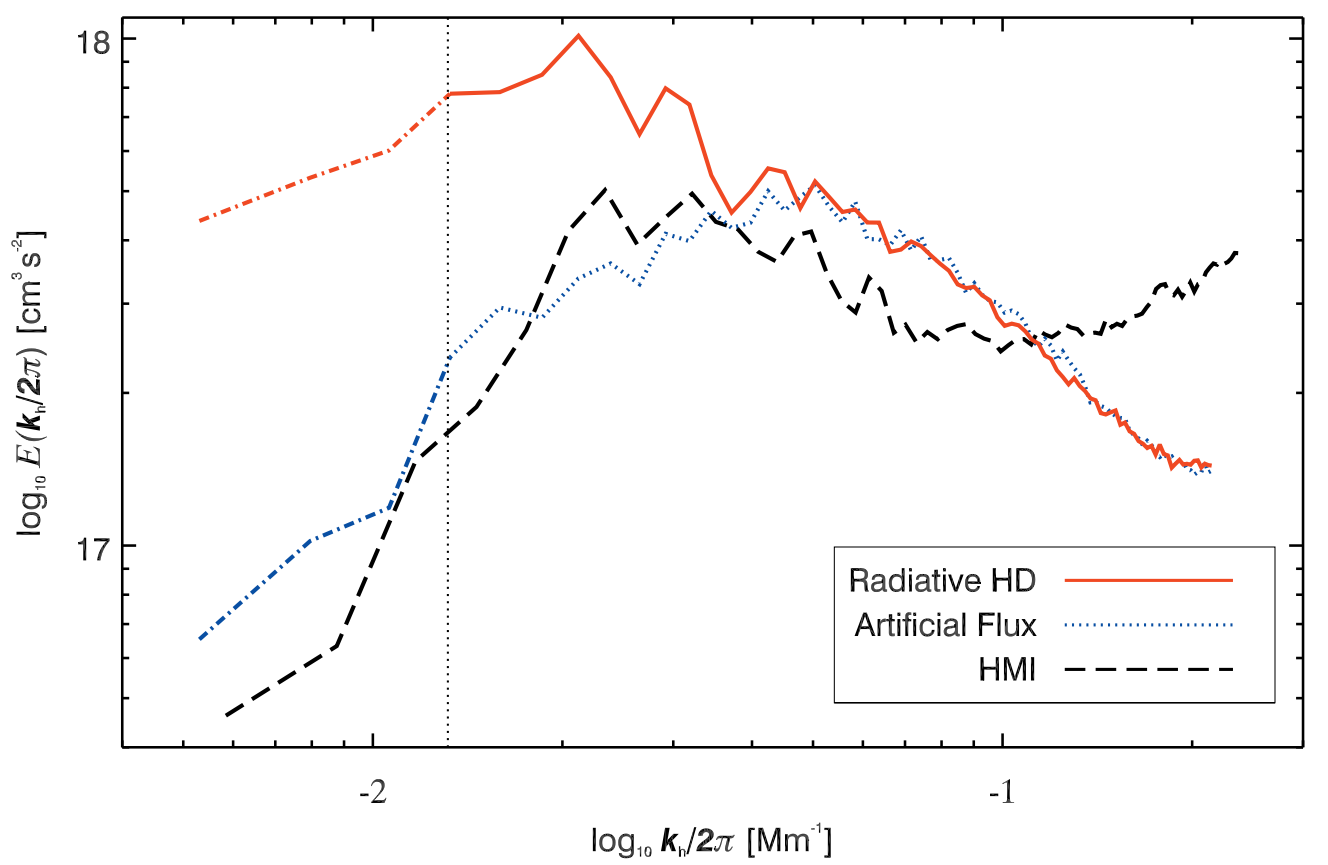

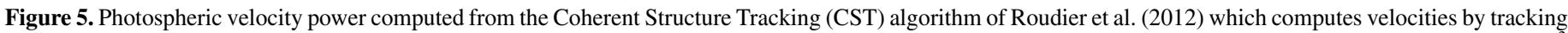

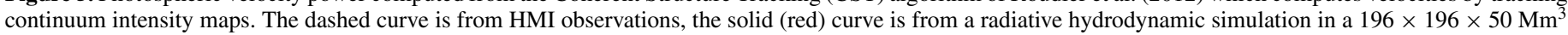

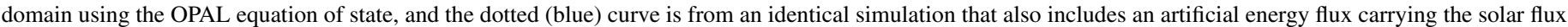

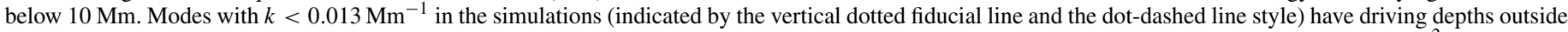

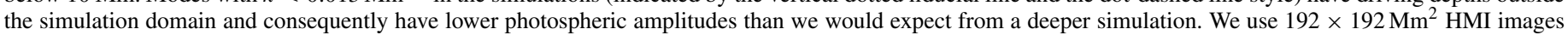

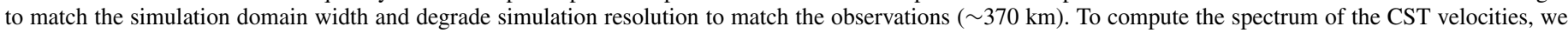

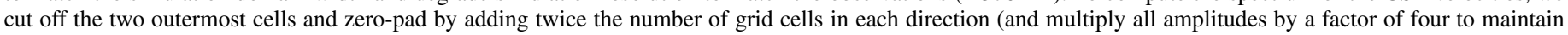
integrated spectral power) to remove the influence of the non-periodic boundary.

(A color version of this figure is available in the online journal.)

decay rate of each mode individually and reducing the overall amplitude of the spectrum by a constant offset factor of two. The increased power in the model spectrum results because the power at the driving depth in the model is overestimated by the assumed Kolmogorov power distribution of the isotropic modes. The factor of two can be removed by using a non-Kolmogorov spectrum at depth, but this introduces additional free parameters that cannot be constrained by solar observations. This highlights an important result: the shape of the horizontal velocity spectrum in the upper layers of the model is largely determined by the vertical velocity amplitude of the modes at depth. The relative amplitudes of large-scale modes in the solar photosphere depend critically on the vertical velocities at the depth. This is further supported by the models' inability to reproduce the simulation results for scales $\lambda_{h}>50 \mathrm{Mm}\left(k / 2 \pi<0.02 \mathrm{Mm}^{-1}\right)$. For these very large scale motions the driving depth lies near the bottom of the simulation domain and the mode amplitudes as well as the measured decay rates are influenced by the simulation lower boundary condition.

\section{SURFACE CONVECTION DEPENDENCE ON MOTIONS AT DEPTH}

The model tests discussed above confirm that the monotonically increasing low wavenumber power and much less prominent supergranular plateau are robust features of the horizontal velocity spectrum. That the model can reproduce the shape of the hydrodynamic simulation spectrum validates the underlying assumption that there are two components to the flow separated by the integral (driving) scale which reflects the local scale height at each depth. Larger scale motions are driven deep in the convection zone and decay from below with height. Smaller scale motions behave as isotropic homogenous turbulence. Mismatches between the model and hydrodynamic simulation spectra and observations, however, raise broader questions. What is the spectrum of solar convective motions at depth and what governs the decay of these motions with height?

\subsection{The Problem of Excess Low Wavenumber Power}

Both the simplified model and the full three-dimensional radiative hydrodynamic spectra show more power than the Sun at scales larger than supergranulation, with that power increasing monotonically toward lower wavenumbers because large-scale flows are convectively driven in the deep layers of the domains. It is worth noting that if the solar spectrum matched either the simplified model or the radiative hydrodynamic simulation spectrum, giant cell convection would be relatively easy to observe as the power in these large-scale modes would exceed that in supergranulation.

To make a more direct comparison between our numerical simulations and observations, we employed a Coherent Structure Tracking (CST; Roudier et al. 2012) algorithm to infer the horizontal velocities on large scales from measurements of the motions of granules. In Figure 5 we compare the CST horizontal velocity spectra of a large-scale radiative hydrodynamic simulation (solid red curve) using the OPAL equation of state (Rogers \& Iglesias 1992) with that of solar observations (dashed black curve) from the Helioseismic and Magnetic Imager (HMI) aboard the Solar Dynamics Observatory. The measurements in both cases employ a $22 \mathrm{hr}$ sequence of continuum intensity images with each HMI image separated by 45 s and each simulation image separated by $\sim 40 \mathrm{~s}$. We break this sequence into 
11 two-hour subsets and use the CST method to compute the velocity for each $2 \mathrm{hr}$ window. The spectrum shown is the average spectrum of those 11 velocity computations. The HMI observations are of a $192 \times 192 \mathrm{Mm}^{2}$ region at disk center with a low magnetic activity on 201019 June. The simulation solution was computed using the MURaM code (Vögler et al. 2005) in a $196 \times 196 \times 49 \mathrm{Mm}^{3}$ domain with $192 \times 192 \times 64 \mathrm{~km}^{3}$ grid spacing (J. W. Lord 2014, in preparation).

We note that the spectra in Figure 5 are truncated at high wavenumbers because the CST method is not reliable for scales smaller than $2.5 \mathrm{Mm}$. Moreover, low wavenumber modes, those with wavenumbers below $k<0.013 \mathrm{Mm}^{-1}$ (indicated by the vertical fiducial line and dot-dashed line style in Figure 5) have length scales larger than the integral (driving) scale at the bottom of the simulation domain and consequently have lower photospheric amplitudes than they would likely have in a deeper simulation. Between these extremes are two notable mismatches between the simulation and observation spectra: the simulation shows an excess of power at low wavenumbers and a deficit of power at high wavenumbers when compared to observations. Our very wide and deep simulations resolve supergranular scale motions well but under-resolve granular motions. This leads to an inferred CST velocity with reduced power at high $k$, a result that is inconsistent with the actual simulation velocities and HMI observations. The excess of low wavenumber power is, on the other hand, a fundamental difference between the resolved motions in the simulation and those in observations and is robust as the CST constrains large-scale motions better than small-scale motions (Roudier et al. 2012). Thus, understanding the observed solar supergranulation spectrum requires understanding the origin of this low wavenumber reduction in power along with any mechanism that may enhance power at supergranular scales.

Our simplified mixing length model suggests that the low wavenumber vertical motions are driven deep in the convection zone and decrease in amplitude toward the surface. The radiative hydrodynamic simulations behave similarly (Section 4), and reducing the vertical flow velocities at depth reduces the low wavenumber horizontal velocity power in the simulated photosphere and improves the match between simulations and observations. We demonstrate this conclusively via simulations in which convective velocities in the deep layers are reduced without changing the mean stratification of the atmosphere (which is also fundamental to the surface spectrum). This was done using an artificial energy transport term. Specifically, we added an artificial flux function to the energy equation that depends only on depth. The artificial flux carries the full solar flux below a specified depth and none of the flux at heights above this. The hyperbolic tangent flux profile employed is $5.12 \mathrm{Mm}$ wide centered at $10 \mathrm{Mm}$ (where $4 H_{\rho} \sim 20 \mathrm{Mm}$ ), effectively supporting radiative losses from the photosphere by depositing the heat where the divergence of the function is nonzero. In Figure 5 (dotted blue curve) we plot the resulting photospheric horizontal velocity spectrum using the same CST method described above. There is substantially reduced power in the photosphere of the artificial flux simulation in those modes that are driven at depths below $\sim 10 \mathrm{Mm}$ (scales larger than $\sim 20 \mathrm{Mm}$ ). This is the region of the domain for which the artificial energy flux is important and consequently convective (rms) velocities are reduced by a factor of $\sim 2.5$.

The artificial energy flux experiment confirms the hypothesis that low wavenumber modes are driven deep in the simulated convection zone and imprint as horizontal flows in the surface layers. The photospheric power spectrum reflects a hierarchy of driving scales with depth even in fully nonlinear radiative hydrodynamic simulations. It also suggests that neither the radiative hydrodynamic solutions nor the simplified model spectrum capture the true dynamics of the solar convection below $\sim 10 \mathrm{Mm}$. In other words, in the Sun, low wavenumber flows carry much less of the convective energy flux or transport the energy at substantially lower velocities than expected based on the simulations or the model. Flow/enthalpy correlations, essential to convective transport, may thus not be correctly captured by hydrodynamic simulations. This may be due to their limited resolution or result from the boundary conditions applied. For example, the open boundary condition commonly employed in radiative hydrodynamic simulations of photospheric convection may smooth perturbations in the inflowing plasma. Alternatively, magnetic fields, not included in the simulations we have discussed in this paper, may maintain flow correlations and allow convective transport on smaller scales or at lower velocities than predicted by purely hydrodynamic models. Preliminary results from magnetized simulations favor this hypothesis, though the underlying mechanisms are still under investigation and the effect so far appears insufficient to explain solar observations (J. W. Lord 2014, in preparation).

\subsection{Helium Ionization Plays a Minor Role}

The small plateau of power at supergranular scales (solid red curve in Figure 4(a) at $k_{h} \sim 0.04 \mathrm{Mm}^{-1}$ ) reflects the role of helium ionization in determining the convective velocities at depth. Superimposed on the horizontal velocity spectrum in Figure 4(a) we have plotted fiducial vertical lines to highlight the integral (driving) scale at the depths of $50 \% \mathrm{He}$ I $(k / 2 \pi=$ $0.1 \mathrm{Mm}^{-1}, 6 \mathrm{Mm}$ depth) and He II $\left(k / 2 \pi=0.03 \mathrm{Mm}^{-1}\right.$, 17.5 Mm depth) ionization. The plateau of supergranular power falls between these two fiducial lines. We have also computed the horizontal velocity spectrum for mixing length background atmospheres with equations of state that do not allow He II or both $\mathrm{He}$ I and He II ionization (Figure 4, dashed (green) and dotted (blue) curves, respectively). These test atmospheres show a continuous power law increase toward low wavenumbers with no feature at supergranular scales. More precisely, these spectra do not show the suppression of power at scales corresponding to the integral scale at the depths of $50 \% \mathrm{He}$ I or He II when the ionization processes are disallowed. The differences between the ionizing and non-ionizing spectra at still lower wavenumbers result because the stratification in the deep layers lies along a different adiabat. The velocity differences at depths below helium ionization, reflected in the low wavenumber horizontal velocity spectra in the near surface, are due to differences in the mean stratification as the medium is nearly fully ionized. In the region of partial ionization, convective velocities are also influenced by the availability of ionization energy in heat transport via perturbations about the mean ionization state.

Helium ionization is thus responsible for the small supergranular plateau in the model spectrum, albeit in a curious fashion. The ionization of helium yields a slight reduction in the driving scale mode amplitudes in the partially ionized regions (where the driving scale $\lambda_{h} \sim 10 \mathrm{Mm}$ for He I and $\lambda_{h} \sim 35 \mathrm{Mm}$ for He II), producing a small apparent enhancement of power in the upper layers at wavenumbers that lie between these driving scales (where $\lambda_{h} \sim 20 \mathrm{Mm}$ ). The reduction in mode amplitudes results because ionization energy contributes to the heat transport. In a partially ionized fluid the heat can be transported by ionization state perturbations as well as thermal perturbations (Rast et al. 1993; Rast \& Toomre 1993), and convective 
velocities in the mixing length model are thus reduced in partially ionized regions. (We note that the mixing length model is a local transport model and does not take into account other effects of ionization such as the increased linear (Rast 1991) and nonlinear (Rast 2001) instability of the fluid, though these may play a role in solar convective flows or in our more complete threedimensional simulations.) The vertical velocity amplitudes of modes that begin their decay at the depths of helium ionization (the integral or driving scale modes at those depths) are thus suppressed, resulting in a reduction in their horizontal velocity power near the surface. Since ionization energy transport depends on ionization state perturbations, with transport in a fully neutral or fully ionized plasma behaving as an ideal gas, modes with peak amplitudes (those with integral scales equal to $4 H_{\rho}$ ) at depths that lie between the partially ionized regions $(10 \mathrm{Mm}$, for example, where $\lambda_{h} \sim 20 \mathrm{Mm}$ and $k / 2 \pi=0.04 \mathrm{Mm}^{-1}$ ) have more power than neighboring modes.

This is illustrated by Figure 4(b), which shows the vertical velocity power at the driving depth (i.e., the depth where the wavelength of the mode is equal to the integral (driving) scale) for mixing length model atmospheres which do or do not allow He I or He II ionization. The driving scale modes in the regions of partial ionization have lower amplitudes than those outside of it, with minima in the mode amplitudes occurring at the depths of $50 \%$ ionization when allowed. The role of hydrogen ionization is difficult to illustrate as its effect is dominant in the surface layers where hydrogen recombination supports radiative losses. The region of $\mathrm{H}$ partial ionization is broad in depth and integral to the structure of the radiative boundary layer, and experiments preventing $\mathrm{H}$ ionization dramatically alter the mean state of the atmosphere and result in dramatic changes in the velocity spectrum across a wide range of wavenumbers. Thus we do not explicitly consider an atmosphere that disallows hydrogen ionization, but it is clear from Figure 4(b) that the effect of hydrogen ionization on mode amplitudes overlaps that of He I (compare crosses (blue) and triangles (green)).

The simple model we have presented thus suggests that there is an apparent enhancement of photospheric power at $\sim 20 \mathrm{Mm}$ scales that occurs because helium ionization reduces the flow speeds in the regions of partial ionization which suppresses power at larger $(\sim 35 \mathrm{Mm}$ from He II $)$ and smaller $(\sim 10 \mathrm{Mm}$ from $\mathrm{He}$ I) scales, not because He II ionization enhances the driving of flows at this depth as has been previously suggested. This apparent enhancement, however, is much smaller than the increased power in observations of solar supergranulation. To investigate the suppression of photospheric power by helium ionization in the context of solar-like convection we use the same Saha equations of state described above in threedimensional radiative hydrodynamic simulations. We use the MURaM (Vögler et al. 2005) code to run simulations that use $192 \times 192 \mathrm{~km}^{2}$ horizontal resolution and $64 \mathrm{~km}$ vertical resolution with $1024 \times 1024 \times 768$ grid cells (giving a domain size of $196 \times 196 \times 49 \mathrm{Mm}^{3}$ ). The results presented here are from more than 5 days of solar time after the simulation has reached a relaxed equilibrium (J. W. Lord 2014, in preparation).

Figure 6(a) shows a comparison of the photospheric horizontal velocity spectrum from three such simulations, one that allows $\mathrm{H}, \mathrm{He}$, and $\mathrm{He}$ II ionization (solid red curve), one in which only $\mathrm{H}$ and $\mathrm{He}$ I ionization are permitted (dashed green curve), and one with only $\mathrm{H}$ ionization (dotted blue curve). The resulting horizontal velocity spectra show suppression of photospheric power similar to that seen in the simplified model (Figure 4) when ionization is allowed. The two lowest wavenumber
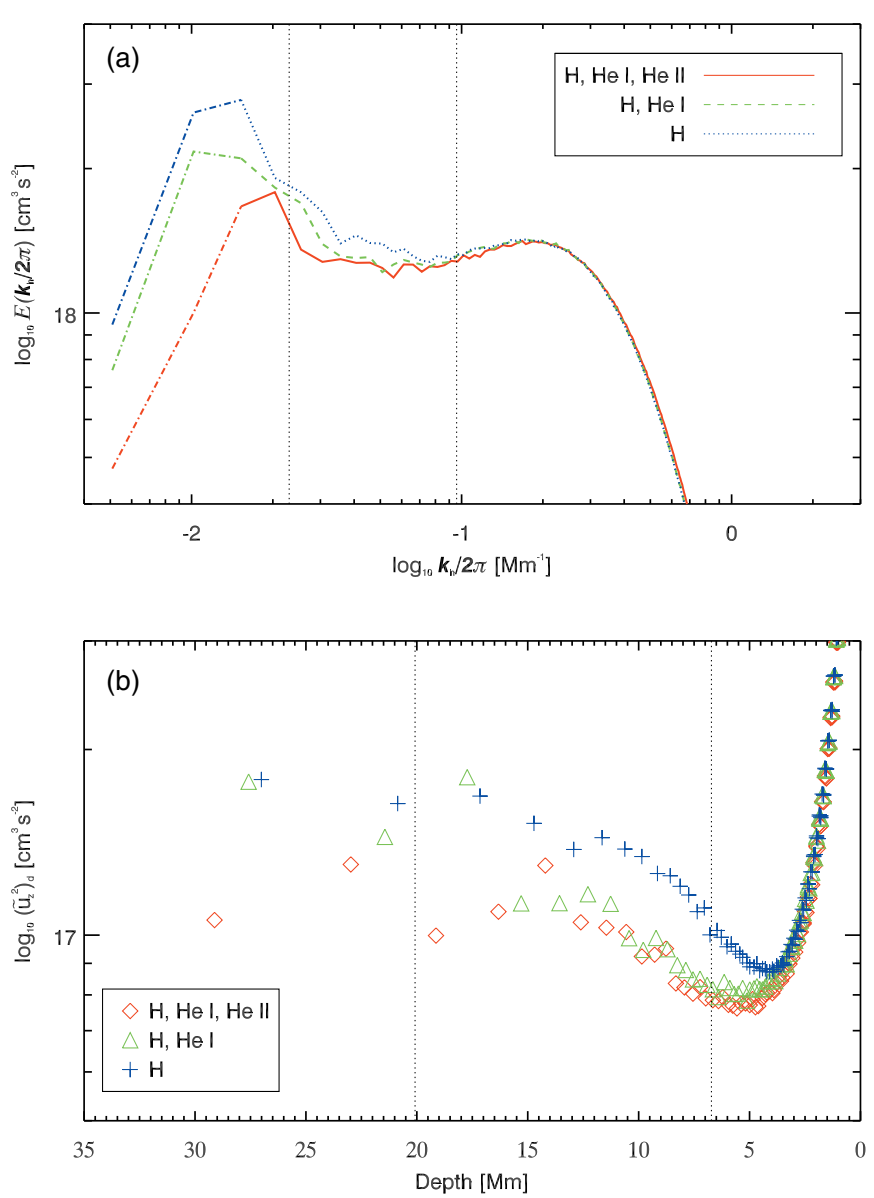

Figure 6. (a) Horizontal velocity spectrum at the photosphere and (b) vertical velocity power at the driving depth. The solid curve in (a) and diamonds in (b) (red) are spectra with a Saha equation of state including H, He I, and He II ionization; the dashed curve in (a) and triangles in (b) (green) use an equation of state with no He II ionization; and the dotted curve in (a) and crosses in (b) (blue) use an equation of state with no He I or II ionization. The vertical dotted lines show the depths of $50 \% \mathrm{He} \mathrm{I}\left(7 \mathrm{Mm}\right.$ and $\left.k / 2 \pi=0.1 \mathrm{Mm}^{-1}\right)$ and He II ( $20 \mathrm{Mm}$ and $k / 2 \pi=0.025 \mathrm{Mm}^{-1}$ ) ionization in (b) and the driving scale, where $\lambda_{h}=4 H_{\rho}$, at those depths in (a). Note that the two largest scale modes are shown with the dot-dashed line style here because the driving depth is outside the simulation domain which makes them unreliable.

(A color version of this figure is available in the online journal.)

modes (dot-dashed line style) have driving depths outside of the simulation domain and are consequently unreliable and weaker than what would be expected in a deeper simulation. The modes with integral (driving) scales equal to $4 H_{\rho}$ in the regions of partial helium ionization again have reduced amplitudes. This is particularly apparent at the wavenumbers corresponding to modes that peak in the He II partial ionization region $(k / 2 \pi$ near $0.013 \mathrm{Mm}^{-1}$ in Figure 6(a)) which is well separated from the effects of hydrogen ionization. Not allowing He II ionization (dashed green and dotted blue curves) causes a small but significant elevation of photospheric power at those wavenumbers. Disallowing He I ionization (dotted blue curve) induces smaller differences due to the dominant role of hydrogen in the surface layers.

The same reduction in the mode amplitude of the vertical velocity spectrum at the driving depths corresponding to partial helium ionization seen in the simplified model is apparent in these hydrodynamic simulations (Figure 6(b)). Modes with scales equal to $4 H_{\rho}$ at the depths of partial He I and He II ionization have reduced amplitudes, though this reduction is 
noisier in the simulation than in the mixing length atmosphere (we note that the rms velocity amplitudes, not shown here, also very clearly increase at the nominal ionization depths when ionization is disallowed). This is due to three primary factors: the spectral resolution of the simulation is limited by the domain width, the three-dimensional simulation is nonlocal which makes using a single depth a poor representation of the vertical velocity power that reaches the surface, and the intrinsic temporal variation in the power of the modes below $\sim 10 \mathrm{Mm}$ is long compared to the 5 days of simulation time. Moreover, other nonlinear effects of ionization may play some role, as discussed above. These experiments do, however, confirm, in the context of fully nonlinear three-dimensional radiative hydrodynamic simulations, two important results of the simplified model: the horizontal velocity spectrum in the photosphere reflects the amplitude of the vertical velocity at depth and the reduced amplitude of vertical velocity in the regions of partial helium ionization plays a minor role in shaping the spectrum of supergranular flows at the surface.

\section{CONCLUSION}

We have constructed a model that computes the horizontal velocity spectrum near the solar surface based on the amplitudes of modes deep in the solar convection zone. The model has three primary features: it is able to match the shape of the photospheric spectrum in three-dimensional radiative hydrodynamic simulations, shows a small supergranular scale enhancement of power at 20-30 Mm, and an excess of power at lower wavenumbers not seen in observations.

We used the model to examine the role that helium ionization plays in shaping the solar photospheric velocity spectrum. We showed that near the depths of $50 \% \mathrm{He}$ I and He II ionization the amplitudes of the vertical motions are reduced because the solar energy flux can be transported at lower velocities due to the contribution of ionization energy. This manifests itself as a suppression of horizontal velocity power in the surface layers at scales neighboring supergranulation $(\sim 35 \mathrm{Mm}$ scales for He II and $\sim 10 \mathrm{Mm}$ scales for He I). We confirmed this effect in threedimensional radiative hydrodynamic simulations that examined convection with and without helium ionization. We conclude that, instead of enhancing a particular flow scale, He I and He II ionization act to highlight supergranular scales by reducing the power in the adjacent modes. This enhancement is, however, smaller in the models than the observed enhancement of solar photospheric power at supergranular scales.

A robust feature of both the model spectrum and the hydrodynamic simulations is an excess of power at low wavenumbers when compared to solar observations. This highlights two uncertainties that require further study. First, we do not know the convective flux spectrum in the deep layers of the Sun. While we took the flow spectrum to be Kolmogorov for all scales below the integral scale, this assumption only approximates the spectrum observed in hydrodynamic simulations, and it may significantly underestimate the role small scale motions play in transporting heat through the solar convection zone. Moreover, the large-scale hydrodynamic simulations also show excess power at large scales compare to the Sun. Preliminary results from similarly large-scale magnetohydrodynamic simulations suggest that magnetic fields may play a role in reducing convective flow speeds or maintaining the correlations required for the energy flux to be carried by smaller scale motions (J. W. Lord 2014, in preparation), but as yet these effects are too small to explain observations. Second, we do not know how the amplitude of the vertical motions decreases with height in the solar convection zone. The decay rates (with height) of low wavenumber modes may be influenced by solar rotation and the near surface shear layer which are not included in our analysis. It is likely that the supergranular excess in the solar power spectrum is largely defined by the observed decrease in power to lower wavenumbers, and has thus been elusive in simulations that show a monotonic increase in power to lower $k$.

The excess low wavenumber power we find in both our simplified model and realistic simulations adds to other recent evidence that large-scale flows deep in the solar convection zone are weaker than previously thought. It supports suggestions that numerical simulations more generally may have difficulty matching solar observations if they are required to carry all of the solar energy flux in the resolved modes ( $\mathrm{N}$. Featherstone 2014, private communication). Helioseismic observations (Hanasoge et al. 2010, 2012) yield estimates of flow velocities that are an order of magnitude or two below those found in either global (e.g., Miesch et al. 2008) or local area (J. W. Lord 2014, in preparation) simulations. Moreover, as global simulations become more turbulent, with lower diffusivities, flow speeds increase and differential rotation profiles flip to an anti-solar configuration, with a slow equator and fast poles because rotational constraints are too weak. This transition to anti-solar behavior can be avoided by decreasing the heat flux through the convection zone or increasing the rotation rate (J. Toomre et al. 2013, private communication; P. Charbonneau 2014, private communication; H. Hotta et al. 2014, in preparation). We found that reducing the convective transport role of large-scale modes (by employing an artificial energy flux at all depths below $10 \mathrm{Mm}$ which reduces the deep rms velocities by a factor of $\sim 2.5$ ) can significantly improved the match between the coherent structure tracking spectra of the simulations and observations. These separate lines of evidence all suggest that the Sun transports energy through the convection zone while maintaining very low amplitude large-scale motions. Something is missing from our current theoretical understanding of solar convection below $\sim 10 \mathrm{Mm}$.

We thank J. Christensen-Dalsgaard for generously providing Model $\mathrm{S}$ mixing length velocities. This work was supported in part by NASA award number NNX12AB35G. R.H.C. acknowledges support from DFG SFB 963 "Astrophysical Flow Instabilities and Turbulence" (Project A1). We acknowledge high-performance computing support from Yellowstone (http://n2t.net/ark:/85065/d7wd3xhc) provided by NCAR's Computational and Information Systems Laboratory, sponsored by the National Science Foundation, and from the NASA High-End Computing (HEC) Program through the NASA Advanced Supercomputing (NAS) Division at Ames Research Center. Computational resources were also provided by NSF-MRI Grant CNS-0821794, MRI-Consortium: Acquisition of a Supercomputer by the Front Range Computing Consortium (FRCC), with additional support from the University of Colorado. NCAR is sponsored by the National Science Foundation.

\section{REFERENCES}

Böhm-Vitense, E. 1958, ZA, 46, 108

Christensen-Dalsgaard, J., Däppen, W., Ajukov, S. V., et al. 1996, Sci, 272, 1286 Crouch, A. D., Charbonneau, P., \& Thibault, K. 2007, ApJ, 662, 715

DeRosa, M. L., \& Toomre, J. 2004, ApJ, 616, 1242

Gough, D. O. 1969, JAtS, 26, 448 
Hanasoge, S. M., Duvall, T. L., \& DeRosa, M. L. 2010, ApJL, 712, L98

Hanasoge, S. M., Duvall, T. L., \& Sreenivasan, K. R. 2012, PNAS, 109, 11928

Hathaway, D. H., Beck, J. G., Bogart, R. S., et al. 2000, SoPh, 193, 299

Hathaway, D. H., Upton, L., \& Colegrove, O. 2013, Sci, 342, 1217

Kolmogorov, A. N. 1941, Dokl. Akad. Nauk SSSR, 30, 301

Leighton, R. B., Noyes, R. W., \& Simon, G. W. 1962, ApJ, 135, 474

Meunier, N., Tkaczuk, R., Roudier, T., \& Rieutord, M. 2007, A\&A, 461, 1141

Miesch, M. S., Brun, A. S., DeRosa, M. L., \& Toomre, J. 2008, ApJ, 673, 557

Mininni, P. D., Alexakis, A., \& Pouquet, A. 2006, PhRvE, 74, 016303

Nordlund, A., Stein, R. F., \& Asplund, M. 2009, LRSP, 6, 2

November, L. J., \& Simon, G. W. 1988, ApJ, 333, 427

November, L. J., Toomre, J., Gebbie, K. B., \& Simon, G. W. 1981, ApJL, 245, L123

Prandtl, L. 1925, Z. angew. Math. Mech., 5, 136

Rast, M. P. 1991, LNP, 388, 179

Rast, M. P. 2001, ApJ, 561, 191
Rast, M. P. 2003, ApJ, 597, 1200

Rast, M. P., Nordlund, Å, Stein, R. F., \& Toomre, J. 1993, ApJL, 408, L53

Rast, M. P., \& Toomre, J. 1993, ApJ, 419, 224

Rieutord, M., Roudier, T., Malherbe, J. M., \& Rincon, F. 2000, A\&A, 357, 1063

Rogers, F. J., \& Iglesias, C. A. 1992, ApJ, 401, 361

Roudier, T., Rieutord, M., Malherbe, J. M., et al. 2012, A\&A, 540, 8

Roudier, T., Rieutord, M., Malherbe, J. M., \& Vigneau, J. 1999, A\&A, 349, 301

Simon, G. W., \& Leighton, R. B. 1964, ApJ, 140, 1120

Sprout, H. C., Nordlund, Å, \& Title, A. M. 1990, ARA\&A, 28, 263

Stein, R. F., Georgobiani, D., Schaffenberger, W., Nordlund, A., \& Benson, D. 2009, in AIP Conf. Proc. 1094, Proc. 15th Cambridge Workshop on Cool Stars, Stellar Systems, and the Sun, ed. E. E. Stempels (Melville, NY: AIP), 764

Ustyugov, S. D. 2010, PhyS, 142, 014031

van Ballegooijen, A. A. 1986, ApJ, 304, 828

Vögler, A., Shelyag, S., Shüssler, M., et al. 2005, A\&A, 429, 335 